\title{
Impacto da implementação da estratégia Semantic Feature Analysis em turmas inclusivas
}

\section{Impact of implementing the Semantic Feature Analysis strategy on inclusive \\ classes}

\author{
Elisabete Verde, Sílvia Oliveira, Anabela Cruz-Santos \\ Instituto de Educação, Universidade do Minho, Centro de Investigação em Educação (CIEd)
}

\begin{abstract}
Resumo
Este estudo quasi-experimental teve por finalidade analisar o impacto da estratégia de ensino-aprendizagem Semantic Feature Analysis (versão em Português Europeu) na promoção do vocabulário, junto de 120 alunos, com e sem NEE, do $1 .^{\circ}$ e $2 .^{\circ}$ ciclos do Ensino Básico, distribuídos por três grupos de ensino. Concluiu-se que a $S F A$ é uma estratégia motivadora, flexível e de fácil aprendizagem, tendo sido mais eficaz que os outros métodos de ensino implementados, contribuído para a promoção da aprendizagem do vocabulário, aquisição de conceitos teóricos e compreensão de textos. A dimensão do efeito foi .87 no $1 .^{\circ}$ ciclo, e .88 no $2 .^{\circ}$ ciclo.
\end{abstract}

Palavras-chave: vocabulário, estratégias de ensinoaprendizagem, Semantic Feature Analysis (SFA), $1 .^{\circ}$ e $2 .^{\circ}$ ciclo do ensino básico.

\begin{abstract}
This quasi-experimental study aimed to analyze the impact of the teaching-learning strategy Semantic Feature Analysis (European Portuguese version) in the promotion of vocabulary, on 120 students, with and without SEN, of the $1^{\text {st }}$ and $2^{\text {nd }}$ cycles of the Basic Education, distributed by three groups of education. It was concluded that $S F A$ is a motivating, flexible and easy-to-learn strategy, having been more effective than other teaching methods implemented, contributing to the promotion of vocabulary learning, the acquisition of theoretical concepts and the understanding of texts. The effect size was .87 in the $1^{\text {st }}$ cycle, and .88 in the $2^{\text {nd }}$ cycle.

Keywords: vocabulary, teaching-learning strategies, Semantic Feature Analysis (SFA), $1^{\text {st }}$ and $2^{\text {nd }}$ cycle of basic education.
\end{abstract}

O conhecimento do vocabulário, para além de ser fundamental nas interações sociais, é um fator determinante para as aquisições escolares (Sim-Sim, 1998), existindo uma forte correlação entre o conhecimento do vocabulário e a compreensão da leitura (Duarte, Colaço, Freitas, \& Gonçalves, 2011; Tannenbaum, Torgesen, \& Wagner, 2006). Os alunos que possuem um conhecimento vocabular restrito e pouco profundo nos primeiros anos escolares, geralmente, mostram maiores dificuldades na compreensão da leitura à medida que progridem na escolaridade, principalmente a partir do $3 .^{\circ}$ ano de escolaridade (Hougen \& Ebbers, 2012), fase em começam a aparecer nos textos mais palavras abstratas e específicas de unidades temáticas, que não são do conhecimento da grande maioria dos alunos (Vaughn \& Bos, 2009). Assim, é fulcral a promoção do desenvolvimento conceptual e lexical das crianças através da implementação de estratégias baseadas na investigação (Denton, Vaughn, Wexler, Bryan, \& Reed, 2012; Vaughn \& Bos, 2009).

A Semantic Feature Analysis-SFA (Pittelman, Heimlich, Berglund, \& French, 1991) é uma das estratégias educativas que a investigação mais referencia para promover eficazmente o desenvolvimento da precisão semântica ao nível da leitura e da escrita (Anders \& Bos, 1986; Blachowicz, Fisher, \& Ogle, 2006; Denton, Vaughn, Wexler, Bryan, \& Reed, 2012; Graves, 2006; Hallahan, Lloyd, Kauffman, Weiss, \& Martinez, 2005; Hougen \& Ebbers, 2012; Jitendra, Edwards, Sacks, \& Jacobson., 2004; Vadasy \& Nelson, 2012; Vaughn \& Bos, 2009). Tem vindo a ser utilizada com sucesso, desde os anos 80, na aprendizagem e no desenvolvimento do vocabulário em diversos contextos da sala de aula: ensino ou revisão de palavras específicas e conceitos-chave das várias disciplinas, compreensão de textos (antes, durante e após a leitura do texto) e atividades que envolvam a leitura e a escrita de textos (Pittelman et al., 1991). Possibilita ainda que os alunos concetualizem os novos conhecimentos e que os relacionem com os seus conhecimentos prévios, envolvendo-os em discussões que estimulam as suas experiências para a obtenção de informações sobre o significado das palavras (Pittelman et al., 1991). Desta forma, os alunos são ativamente envolvidos na aprendizagem dos significados das palavras em vez de serem recetores passivos de informação, ao integrarem o seu conhecimento prévio com novas informações e ao construírem categorias semânticas relacionando palavras e conceitos (Blachowicz et al., 2006; Greenwood, 2002), atingindo uma compreensão mais profunda das palavras e, 
consequentemente, uma compreensão mais eficaz do que leem (Vaughn \& Bos, 2009).

A implementação da estratégia SFA implica a construção de uma tabela de dupla entrada (ver Figura 1), tendo por base um sujeito ou um conceito, para organizar conexões entre palavras e ajudar na análise de semelhanças e diferenças entre conceitos relacionados (Pittelman et al., 1991), seguindo-se um processo de sete etapas:

1. Selecionar uma categoria;

2. Listar palavras relacionadas com a categoria;

3. Listar e adicionar características;

4. Determinar as características de pertença e não pertença;

5. Acrescentar mais palavras ou características;

6. Completar a tabela.

7. Analisar e discutir a tabela.

Segundo Pittelman et al. (1991), estas sete etapas descrevem o procedimento básico para utilizar a estratégia $S F A$, em que os conceitos representados por palavras relacionadas com o mesmo são classificados de acordo com as suas características, isto é, cada palavra da coluna da esquerda é analisada característica por característica, colocando-se o sinal mais $(+)$ ou o sinal menos (-) dependendo da sua aplicabilidade, podendo-se usar o ponto de interrogação (?) no caso de surgirem dúvidas. Durante este procedimento, os alunos envolvem-se em discussões, que é primordial para o sucesso desta estratégia (Vaughn \& Bos, 2009). Para estas autoras, a chave de uma discussão frutífera é incentivar os alunos a perguntar uns aos outros a razão da sua classificação da relação entre palavras/conceitos, visto que é uma maneira de encorajá-los a ativar o que já sabiam sobre os vocábulos e, ao justificar os seus raciocínios, pensam sobre os conceitos e relacionam mais eficazmente a informação nova com a antiga, apreendendo de forma mais consistente o significado das palavras.

De acordo com Pittelman et al. (1991), a aula poderá acabar após os alunos terem analisado e discutido a tabela completa, ou poderá servir como uma ferramenta de motivação para uma maior exploração e estudo, dado que, muitos professores descobriram que os alunos ao examinarem a tabela mostraram interesse em aprender mais sobre os conceitos.

Inicialmente, o professor tem um papel fundamental na modelação do processo, explicando aos alunos como devem analisar as palavras ou conceitos através das características, focando as diversas relações entre as palavras (Pittelman et al., 1991). Quando os alunos estiverem familiarizados com o processo, o professor passa a ter um papel facilitador, orientando apenas a análise, discussão e conclusão da tabela (Pittelman et al., 1991). À medida que os alunos se vão tornando proficientes nesta atividade, serão capazes de assumir um papel mais ativo no planeamento da organização da tabela e na escolha das palavras e características a serem comparadas (Pittelman et al., 1991).

\section{Método}

\section{Participantes}

A amostra é constituída por 120 alunos, 65 alunos (5 dos quais com NEE) do $1 .^{\circ}$ ciclo e 55 alunos (sete com NEE) do 2. ${ }^{\circ}$ ciclo do Ensino Básico de escolas de agrupamentos da Região Norte de Portugal.

Os alunos do $1 .^{\circ}$ ciclo, com idades compreendidas entre os 7 e os 9 anos $(M=8.06, D P=.35)$, sendo $52.3 \%$ do género feminino e $47.7 \%$ do género masculino, foram distribuídos por três grupos: grupo de ensino regular (GER), grupo de ensino estruturado (GEE) e grupo de ensino da estratégia SFA (GESFA).

Os alunos do $2 .^{\circ}$ ciclo ( $43.6 \%$ do género feminino e $56.4 \%$ do género masculino), com idades compreendidas entre os 10 e os 13 anos $(\mathrm{M}=10.47, \mathrm{DP}=.74)$, foram distribuídos por três grupos: grupo de controlo (GC), grupo escola virtual (GEV) e grupo experimental (GE).

\section{Instrumentos}

Foi utilizada uma ficha de verificação de conhecimentos de acordo com o ciclo de ensino, aplicada em dois momentos distintos, de pré e pós-teste, a todos os participantes.

A ficha de verificação de conhecimentos para o $1 .^{\circ}$ ciclo foi elaborada com base no estudo de Johnson, Pittelman, Toms-Bronowski, e Levin (1984), com o objetivo de avaliar os conhecimentos dos alunos relativamente à compreensão e aplicação de vocabulário/conceitos teóricos relacionados com o tema "Os animais", no âmbito das disciplinas de Português e de Estudo do Meio, do $3 .^{\circ}$ ano de escolaridade. Era constituída por um texto informativo e oito questões (escolha múltipla, verdadeiro/falso, preenchimento de lacunas, crucigrama, completamento, associação), com diferentes graus de dificuldade, e distribuídas por dois grupos: compreensão do texto (Grupo I) e aplicação de vocabulário (Grupo II).

A ficha de verificação de conhecimentos para o $2 .^{\circ}$ ciclo foi elaborada a partir do estudo de Toms-Bronowski (1982), com o intuito de verificar os conhecimentos dos alunos relativamente ao vocabulário inerente ao tema "Diversidade dos animais" no âmbito da disciplina Ciências Naturais do 5. ${ }^{\circ}$ ano. Era constituída por 30 itens (escolha múltipla, verdadeiro/falso, relacionação, preenchimento de lacunas e crucigrama), divididos por cinco questões introduzidas por um pequeno texto explicativo.

Também se aplicou um questionário de satisfação aos alunos e aos professores dos grupos onde foi implementada a estratégia $S F A$, com o intuito de aferir o grau de satisfação destes relativamente à estratégia.

$\mathrm{Na}$ implementação da estratégia $S F A$ utilizaram-se tabelas $S F A$ (ver Figuras 1 e 2). 


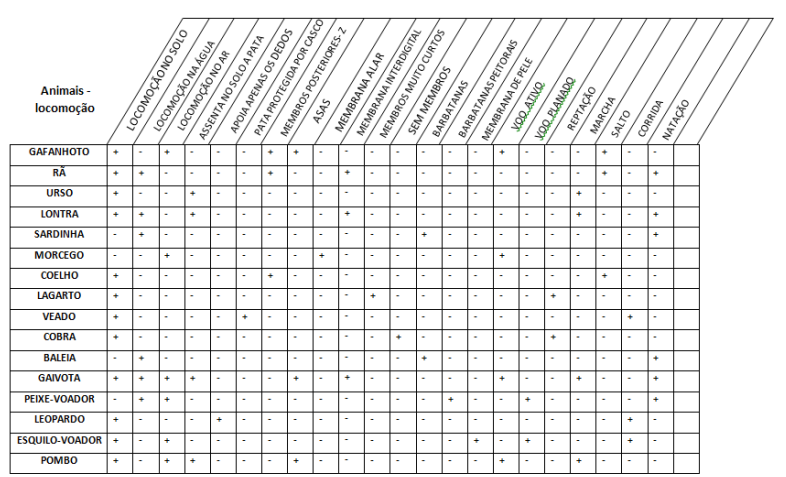

Figura 1. Exemplo completo da tabela da estratégia $S F A$ sobre o conceito: locomoção dos animais (1. ${ }^{\circ}$ ciclo).

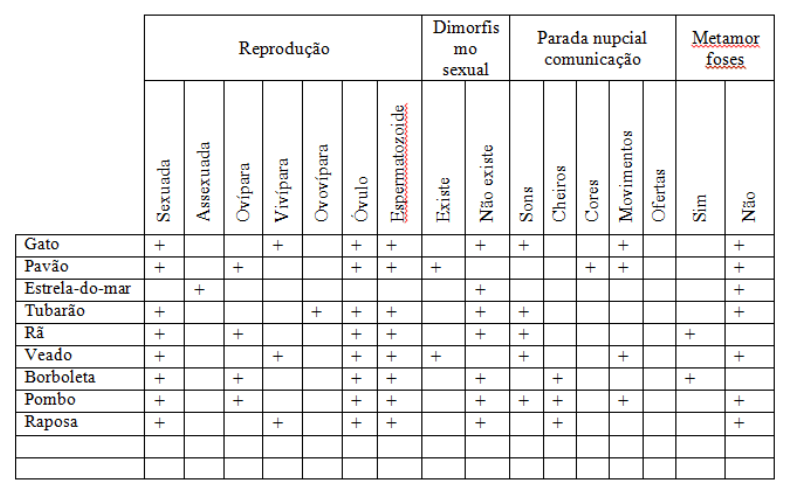

Figura 2. Exemplo completo da tabela da estratégia $S F A$ sobre o conceito: reprodução dos animais (2. ${ }^{\circ}$ ciclo).

\section{Procedimentos}

Numa primeira fase, procedeu-se à tradução e adaptação da estratégia $S F A$ para Português Europeu. Seguidamente, foi pedida a autorização e consentimento aos presidentes dos conselhos pedagógicos dos respetivos agrupamentos, aos diretores de turma e aos professores da disciplina de Ciências Naturais (2. ${ }^{\circ}$ ciclo), aos professores titulares de turma $\left(1 .^{\circ}\right.$ ciclo), pais e encarregados de educação.

Foi implementado um estudo piloto com a finalidade de aferir a exequibilidade da aplicabilidade da ficha de verificação de conhecimentos, junto de alunos não pertencentes à amostra, que frequentavam o mesmo agrupamento dos alunos que faziam parte da investigação, mas provenientes de outro estabelecimento de ensino.

No passo seguinte, procedeu-se à aplicação da ficha de verificação de conhecimentos, no momento de pré-teste, em todos os grupos da amostra, garantindo todas as condições para que os alunos a pudessem realizar sem constrangimentos.

Terminada a fase de aplicação de pré-teste, iniciou-se a implementação da estratégia $S F A$ nos grupos GESFA (1. ${ }^{\circ}$ ciclo) e GE (2. ${ }^{\circ}$ ciclo) para aquisição de vocabulário e compreensão de conceitos teóricos, ao longo de dez sessões, cada uma com a duração entre 45 e 60 minutos. No início as sessões foram controladas pela investigadora, explicitando todos os passos e procedimentos a seguir na implementação da estratégia. As tabelas $S F A$ utilizadas foram inicialmente preenchidas pelos alunos em conjunto com a investigadora, depois pelos alunos em pequeno grupo/pares e, mais tarde, de forma individual.

As primeiras tabelas apresentavam toda a informação que se pretendia conhecer e à medida que se foi avançando na aprendizagem, estas foram aparecendo com cada vez com menos informação até serem apresentadas totalmente em branco. Nesta fase, com a ajuda da investigadora, os alunos colocavam as palavras/categorias que interessava conhecer e as características que poderiam possuir. Seguidamente, os alunos construíam a sua tabela de forma individual e, no final, discutiam-se os resultados.

Os restantes grupos: GER e GEE (1. ${ }^{\circ}$ ciclo) e GC e GEV (2. ${ }^{\circ}$ ciclo) foram intervencionados em simultâneo, mas com recurso a metodologias diferentes. No 1. ${ }^{\circ}$ ciclo, os alunos do GER continuaram a beneficiar de um ensino regular (procura no dicionário, memorização de definições e escrita de frases) usado habitualmente pelo professor titular e realizando as atividades que constavam nos seus manuais. Por sua vez, o GEE usufruiu de uma aprendizagem do vocabulário relacionado com o tema "Os animais", por parte do professor titular de turma, através da realização das atividades dos manuais e fichas de trabalho, da construção de cartazes (exemplos e não exemplos de palavras) e mapas semânticos, e trabalhou a compreensão e interpretação de textos recorrendo a diversas estratégias (e.g., procura no dicionário, análise contextual, análise morfológica).

No 2. ${ }^{\circ}$ ciclo, o GC beneficiou de um ensino regular ministrado pelo professor da disciplina recorrendo ao manual e à realização de fichas de trabalho do mesmo. $\mathrm{O}$ professor do GEV recorreu a filmes e descrições existentes na plataforma Escola Virtual. Estes alunos respondiam a questões sobre a matéria em estudo de forma interativa.

Após terminada a fase da implementação da estratégia e a intervenção nos restantes grupos, todos os alunos que constituíam a amostra realizaram a ficha de verificação de conhecimentos no momento de pós-teste, tendo-se aplicado os mesmos procedimentos do momento de préteste. A finalidade foi aferir o desempenho de todos os alunos, após as intervenções, relativamente à aquisição e compreensão de vocabulário/conceitos e conhecer o impacto produzido pela implementação da estratégia SFA.

Finalmente, foram aplicados os questionários de forma a aferir o grau de satisfação dos alunos e professores dos grupos GESFA (1. ${ }^{\circ}$ ciclo) e GE (2. ${ }^{\circ}$ ciclo).

\section{Resultados}

No $1 .^{\circ}$ ciclo, o teste de Kruskal-Wallis indicou que os resultados obtidos pelos alunos relativos à compreensão do texto variam em função do grupo em que estão inseridos (ver Tabela 1). Os testes de Mann-Whitney com correção de Bonferroni revelaram que existem diferenças estatisticamente significativas, no pré e no pós-teste, entre o GESFA e o GER (pré-teste: $U=115.50, p=.001$, $r=-.48$; pós-teste: $U=53.50, p<.001, r=-.67)$ e entre o GESFA e o GEE (pré-teste: $U=140.50, p=.011, r=-.37$; pós-teste: $U=42.50, \quad p<.001, r=-.68)$, no entanto, as 
diferenças entre o GER e o GEE não são estatisticamente significativas.

Tabela 1.

Diferenças entre os grupos relativamente à compreensão do texto, no pré e no pós-teste

\begin{tabular}{|c|c|c|c|c|}
\hline & $\begin{array}{c}\text { GER } \\
(n=18) \\
\text { Ordem } \\
\text { média }\end{array}$ & $\begin{array}{c}\text { GEE } \\
(n=17) \\
\text { Ordem } \\
\text { média }\end{array}$ & $\begin{array}{c}\text { GESFA } \\
(n=30) \\
\text { Ordem } \\
\text { média }\end{array}$ & $H(2)$ \\
\hline $\begin{array}{l}\text { Pré- } \\
\text { teste }\end{array}$ & 42.86 & 38.38 & 24.03 & $13.03 * *$ \\
\hline $\begin{array}{l}\text { Pós- } \\
\text { teste }\end{array}$ & 22.31 & 19.09 & 47.30 & $32.20 * * *$ \\
\hline & 7 & & & \\
\hline
\end{tabular}

No que se refere à aplicação de vocabulário, constatouse a existência de diferenças estatisticamente significativas (ver Tabela 2), quer no pré quer no pósteste, entre o GESFA e o GER (pré-teste: $U=159.50$, $p=.018, r=-.34$; pós-teste: $U=20.50, p<.001, r=-.77) \mathrm{e}$ entre o GER e o GEE (pré-teste: $U=72.50, p=.007, r=-$ .45 ; pós-teste: $U=80.00, p=.016, r=-.41$ ). O GESFA e o GEE não apresentaram diferenças estatisticamente significativas no pré-teste, contudo, após a implementação da estratégia $S F A$, observou-se a sua existência $(U=111.00, p=.001, r=-.47)$.

Tabela 2.

Diferenças entre os grupos relativamente à aplicação de vocabulário, no pré e no pós-teste

\begin{tabular}{|c|c|c|c|c|}
\hline & $\begin{array}{c}\text { GER } \\
(n=18) \\
\text { Ordem } \\
\text { média }\end{array}$ & $\begin{array}{c}\text { GEE } \\
(n=17) \\
\text { Ordem } \\
\text { média }\end{array}$ & $\begin{array}{c}\text { GESFA } \\
(n=30) \\
\text { Ordem } \\
\text { média }\end{array}$ & $H(2)$ \\
\hline $\begin{array}{l}\text { Pré- } \\
\text { teste }\end{array}$ & 43.61 & 27.59 & 29.70 & $8.05 *$ \\
\hline $\begin{array}{l}\text { Pós- } \\
\text { teste }\end{array}$ & 15.08 & 28.82 & 46.12 & $31.97 * * *$ \\
\hline$* p<.05$ & $* * * p$ & & & \\
\hline
\end{tabular}

O teste de Wilcoxon (ver Tabela 3) indicou que existem diferenças estatisticamente significativas no GESFA, entre o pré e o pós-teste, relativamente à compreensão do texto e à aplicação de vocabulário, cuja dimensão do efeito é de .87. Nos grupos GER e GEE só se verificaram diferenças estatisticamente significativas no que respeita à aplicação de vocabulário, contudo quando comparados com o GESFA foram os alunos da estratégia $S F A$ que apresentaram resultados superiores.

Tabela 3.

Comparação entre os resultados obtidos no pré e os resultados obtidos no pós-teste

\begin{tabular}{cccc}
\hline & $\begin{array}{c}\text { GER } \\
(n=18)\end{array}$ & $\begin{array}{c}\text { GEE } \\
(n=17)\end{array}$ & $\begin{array}{c}\text { GESFA } \\
(n=30)\end{array}$ \\
& $Z$ & $Z$ & $Z$ \\
\hline $\begin{array}{c}\text { Compreensão } \\
\text { do texto }\end{array}$ & - & - & $-4.78 * * *$ \\
\hline
\end{tabular}

\begin{tabular}{llll}
\hline $\begin{array}{c}\text { Aplicação de } \\
\text { vocabulário }\end{array}$ & $-3.42 * *$ & $-3.63 * * *$ & $-4.79 * * *$ \\
\hline$* * p<.01 \quad * * * p<.001$ & & \\
\hline & &
\end{tabular}

No 2. ${ }^{\circ}$ ciclo, os resultados demonstraram que o desempenho dos alunos no pré-teste não varia em função do grupo em que estão inseridos, $H(2)=4.25, p=.119$, no entanto, no pós-teste, o teste de Kruskal-Wallis detetou diferenças estatisticamente significativas entre os grupos, $H(2)=20.08, p<.001$ (ver Tabela 4). Os testes de Mann-Whitney com correção de Bonferroni evidenciaram que há diferenças estatisticamente significativas entre o grupo GEV e GE $(U=48.50, p<$ $.001, r=-.64)$ e entre os grupos GC e GE ( $U=49.00, p$ $<.001, r=-.59)$. Contudo, a análise revela que as diferenças entre os grupos GEV e GC não são estatisticamente significativas $(U=121.00, p=.305)$.

Tabela 4.

Diferenças entre os grupos em estudo ao nível do pósteste

\begin{tabular}{lcccc}
\hline & GEV & GC & GE & \\
& $(n=19)$ & $(n=16)$ & $(n=20)$ & \\
& Ordem & Ordem & Ordem & $H(2)$ \\
& média & média & média & \\
\hline $\begin{array}{l}\text { Pós- } \\
\text { teste }\end{array}$ & 18.92 & 23.00 & 40.63 & $20.08 * * *$ \\
\hline$* * * p<.001$ & & & \\
\hline
\end{tabular}

Aplicou-se o teste de Wilcoxon para aferir se o desempenho dos alunos difere ou não nos dois momentos avaliativos, indicando um aumento no desempenho dos alunos do pré-teste para o pós-teste (ver Figura 3), $Z=$ $3,92 p<.001, r=-.88$, do GE.

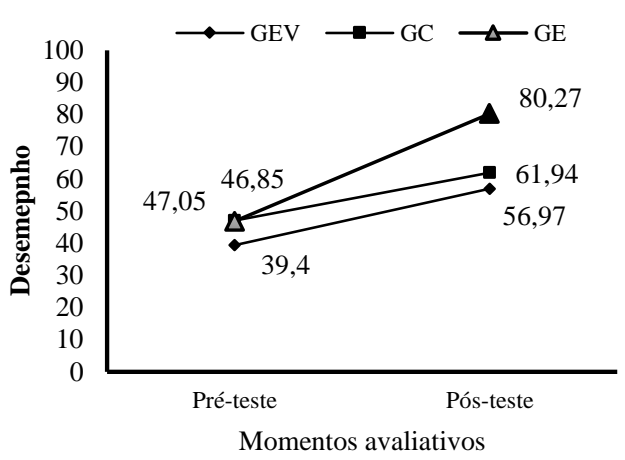

Figura 3. Comparação entre os resultados obtidos no pré e no pós-teste.

Como se pode observar na Tabela 5, todos os alunos com Necessidades Educativas Especiais (NEE) evoluíram positivamente do pré para o pós-teste, contudo foram os alunos dos grupos onde foi implementada a estratégia $S F A$ que apresentaram uma maior evolução na sua aprendizagem.

Tabela 5 .

Resultados obtidos pelos alunos com NEE, nos momentos de pré e pós-teste 


\begin{tabular}{|c|c|c|c|c|}
\hline & Grupos & $\begin{array}{c}\text { Alunos com } \\
\text { NEE }\end{array}$ & $\begin{array}{c}\text { Pré-teste } \\
(\%)\end{array}$ & $\begin{array}{c}\text { Pós-teste } \\
(\%)\end{array}$ \\
\hline \multirow{5}{*}{\begin{tabular}{l}
$\frac{0}{0}$ \\
\hdashline \\
$\stackrel{0}{\circ}$ \\
$\circ$
\end{tabular}} & GER & Aluno A & 35.00 & 41.00 \\
\hline & & Aluno B & 20.50 & 38.00 \\
\hline & GEE & Aluno C & 26.50 & 42.50 \\
\hline & & Aluno D & 24.00 & 88.00 \\
\hline & GESFA & Aluno E & 28.50 & 83.00 \\
\hline \multirow{7}{*}{ 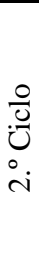 } & & Aluno F & 7.80 & 35.20 \\
\hline & GEV & Aluno G & 40.22 & 43.00 \\
\hline & & Aluno $\mathrm{H}$ & 50.00 & 54.00 \\
\hline & GC & Aluno I & 24.40 & 47.20 \\
\hline & \multirow{3}{*}{ GE } & Aluno J & 48.00 & 81.80 \\
\hline & & Aluno K & 44.60 & 81.40 \\
\hline & & Aluno L & 48.00 & 86.80 \\
\hline
\end{tabular}

Em suma, a implementação da estratégia $S F A$ nos grupos experimentais foi responsável pela superioridade destes grupos em relação aos restantes.

\section{Discussão}

Os resultados obtidos levam-nos a concluir que a estratégia SFA é motivadora, apelativa, pouco dispendiosa, flexível e de fácil aplicação. O seu processo de aprendizagem é simples e facilmente compreendido pelos alunos, podendo ser ensinada, com sucesso, em sala de aula inclusiva, nas várias disciplinas e em todos os anos de escolaridade, corroborando assim com os estudos de Anders, Bos e Filip (1984) e Jitendra et al. (2004), dado que todos os alunos, sem e com NEE, aprenderam a estratégia, compreenderam o seu procedimento e a sua utilidade e melhoraram significativamente $o$ seu desempenho.

A estratégia $S F A$ foi mais eficaz que as estratégias implementadas nos outros grupos da amostra, em que os resultados indicaram uma real evolução positiva entre o pré e o pós-teste, cuja dimensão do efeito indicou um valor de .87 no $1 .^{\circ}$ ciclo e de .88 no $2 .^{\circ}$ ciclo. O sucesso obtido pelos alunos neste estudo é comprovado por outros estudos realizados por Anders et al. (1986), Johnson et al. (1984) e de Toms-Bronowski (1982), que demonstram um maior sucesso dos alunos na aprendizagem de vocabulário e compreensão da leitura após a aplicação da estratégia $S F A$. Efetivamente, tal como afirmam Denton et al. (2012), Jitendra et al. (2004), Pittelman et al. (1991) e Vadasy e Nelson (2012), a SFA é uma estratégia versátil para o desenvolvimento do vocabulário, podendo ser usada para o aumentar, refinar ou reforçar nas áreas temáticas ou para ensinar palavras específicas que representam conceitos-chave na compreensão do texto que será lido.

No final do estudo, os alunos revelaram, na sua totalidade, gostar de aprender a estratégia $S F A$, e os professores mostraram-se satisfeitos com a implementação e eficácia da estratégia $S F A$. Estas opiniões são corroboradas por Pittelman et al. (1991), que referem que os alunos sentem-se mais motivados e com mais confiança nas suas capacidades, dado que a implementação da $S F A$ promove um ambiente cooperativo na sala de aula e faz com que se envolvam ativamente na aprendizagem de palavras/conceitos, ajudando-os a compreender e a visualizar as relações entre palavras, aumentando assim a capacidade de retenção dos significados de novas palavras e de efetuar a sua rechamada.

\section{Referências}

Anders, P. L., \& Bos, C. S. (1986). Semantic Feature Analysis: An Interactive Strategy for Vocabulary Development and Text Comprehension. Journal of Reading, 29, 610-616.

Anders, P. L., Bos, C. S., \& Filip, D. (1984). The effect of semantic feature analysis on the reading comprehension of learning-disabled students. In J. S. Niles and L. A. Harris (Eds.), Changing Perspectives on Reading/Language Processing and Instruction (pp. 162-166). Rochester, NY: The National Reading Conference.

Blachowicz, C., Fisher, P., \& Ogle, D. (2006). Vocabulary: Questions from the classroom. Reading Research Quarterly, 41(4), 524-539.

Denton, C. A., Vaughn, S., Wexler, J., Bryan, D., \& Reed, D. (2012). Effective instruction for middle school students with reading difficulties: the reading teacher's sourcebook. Baltimore: Paul H. Brookes Publishing Co.

Duarte, I., Colaço, M., Freitas, M., \& Gonçalves, A. (2011). O Conhecimento da Língua: Desenvolver a Consciência Lexical. Programa Nacional do Ensino do Português. Lisboa: Ministério da Educação, DireçãoGeral de Inovação e de Desenvolvimento Curricular.

Graves, M. F. (2006). Instruction on individual words: one size does not fit all. In A. E. Farstrup and S. J. Samuels (Eds.), What research has to say about vocabulary instruction (pp. 56-79). Newark, DE: International Reading Association.

Greenwood, S.C.(2002). Making words matter: Vocabulary study in the content areas. The Clearing House, 75(5), 258-264.

Hallahan, D. P., Lloyd, J. W., Kauffman, J. M., Weiss, M. P., \& Martinez, E. A. (2005). Introduction to learning disabilities: Foundations, characteristics, and effective teaching. Boston: Allyn and Bacon.

Hougen, M. C., \& Ebbers, S. C. (2012). A Comprehensive, Interactive Approach to Vocabulary Development. In M. C. Hougen and S. M. Smartt (Eds.), Fundamentals of Literacy Instruction and Assessment, Pre-K-6 (pp. 150-183). Baltimore: Paul H. Brookes Publishing Co.

Jitendra, A. K., Edwards, L. L., Sacks, G., \& Jacobson, L. A. (2004). What research says about vocabulary instruction for students with learning disabilities. Exceptional Children, 70, 299-322.

Johnson, D. D., Pittelman, S. D., Toms-Bronowski, S., \& Levin, K. M. (1984). An investigation of the effects of prior knowledge and vocabulary acquisition on passage comprehension (Program Report 84-5). Madison, WI: University of Wisconsin, Center for Education Research.

Pittelman, S. D., Heimlich, J. E., Berglund, R. L., \& French, M.P. (1991). Semantic Feature Analysis: Classroom Application. Newark, DE: International Reading Association. 
Sim-Sim, I. (1998). Desenvolvimento da linguagem. Lisboa: Universidade Aberta.

Tannenbaum, K. R., Torgesen, J. K., \& Wagner, R. K. (2006). Relationships between word knowledge and reading comprehension in third-grade children. Scientific Studies of Reading, 10, 381-398.

Toms-Bronowski, S. (1982). An investigation of the effectiveness of semantic mapping and semantic feature analysis with intermediate grade level students (Program Report 83-3). Madison, WI: University of Wisconsin, Center for Education Research.

Vadasy, P. F., \& Nelson, J. R. (2012). Vocabulary instruction for Struggling Students. New York: The Guilford Press.

Vaughn, S. R., \& Bos, C. S. (2009). Strategies for teaching students with learning and behavior problems. New Jersey: Pearson. 